\title{
THE SUB-GAUSSIAN NORM OF A BINARY RANDOM VARIABLE
} UDC 519.21

\author{
V. V. BULDYGIN AND K. K. MOSKVICHOVA
}

\begin{abstract}
The exact values of the sub-Gaussian norms of Bernoulli random variables and binary random variables are found. Exponential bounds for the distributions of sums of centered binary random variables are studied for both cases of independent and dependent random variables. These bounds improve some known results.
\end{abstract}

\section{INTRODUCTION}

The aim of this paper is to find the exact values of sub-Gaussian norms for centered Bernoulli and binary random variables. It is assumed below that all the random variables are defined on the same probability space $\{\Omega, \mathfrak{F}, \mathrm{P}\}$.

A random variable $\xi$ is called sub-Gaussian if there exists a number $a \in[0, \infty)$ such that

$$
\mathrm{E} \exp \{\lambda \xi\} \leq \exp \left\{\frac{a^{2} \lambda^{2}}{2}\right\}
$$

for all $\lambda \in \mathbf{R}=(-\infty, \infty)$.

The number

$$
\tau(\xi)=\inf \left\{a \geq 0: \operatorname{E} \exp \{\lambda \xi\} \leq \exp \left\{\frac{a^{2} \lambda^{2}}{2}\right\}, \lambda \in \mathbf{R}\right\}
$$

is called the sub-Gaussian standard of the random variable $\xi$. A random variable $\xi$ is sub-Gaussian if and only if $\tau(\xi)<\infty$. In this case,

$$
\mathrm{E} \exp \{\lambda \xi\} \leq \exp \left\{\frac{\tau^{2}(\xi) \lambda^{2}}{2}\right\}, \quad \lambda \in \mathbf{R} .
$$

Sub-Gaussian random variables are introduced by Kahane [16. Properties and applications of sub-Gaussian random variables are studied by many authors (see, for example, [1, 4, 7, 9, 13, 16, 17]).

Sub-Gaussian random variables $\xi$ are centered, that is, $\mathrm{E} \xi=0$, and are such that

$$
\mathrm{E} \xi^{2} \leq \tau^{2}(\xi)
$$

The sub-Gaussian standard $\tau(\xi)$ is a norm (called the sub-Gaussian norm). The family of all sub-Gaussian random variables $S u b(\Omega)$ is a Banach space with respect to the norm $\tau(\xi)$ (see [1 $\left[\begin{array}{l}3 \\ ]\end{array}\right)$. If $\tau^{2}(\xi)=\mathrm{E} \xi^{2}$, then $\xi$ is called a strongly sub-Gaussian random variable.

2010 Mathematics Subject Classification. Primary 60G50, 65B10, 60G15; Secondary 40A05.

Key words and phrases. Sub-Gaussian random variable, sub-Gaussian norm, exponential inequalities, sums of random variables, Bernstein inequality, Hoeffding inequality. 
An important property of the sub-Gaussian norm is that the norm of a sum of independent random variables does not exceed the sum of norms of the individual terms, that is,

$$
\tau^{2}\left(\sum_{j=1}^{n} \xi_{j}\right) \leq \sum_{j=1}^{n} \tau^{2}\left(\xi_{j}\right)
$$

for independent sub-Gaussian random variables $\xi_{1}, \ldots, \xi_{n}$.

The distributions of sub-Gaussian random variables admit exponential bounds; see 2 , 3, 9, 16, 17. If $\xi \in \operatorname{Sub}(\Omega)$, then

$$
\begin{gathered}
\mathrm{P}\{\xi \geq x\} \leq \exp \left\{-\frac{x^{2}}{2 \tau^{2}(\xi)}\right\}, \\
\mathrm{P}\{\xi \leq-x\} \leq \exp \left\{-\frac{x^{2}}{2 \tau^{2}(\xi)}\right\}, \\
\mathrm{P}\{|\xi| \geq x\} \leq 2 \exp \left\{-\frac{x^{2}}{2 \tau^{2}(\xi)}\right\}
\end{gathered}
$$

for all $x>0$. Here and in what follows we agree that $\exp \left\{-\frac{1}{0}\right\}=0$.

Every centered Gaussian random variable is sub-Gaussian and the square of its subGaussian norms is equal to the variance. It is known (see [3, 9]) that if $\xi$ is a centered random variable and $\mathrm{P}\{|\xi| \leq c\}=1$, where $c>0$, then $\xi$ is sub-Gaussian and $\tau(\xi) \leq c$. In general, the exact values of the sub-Gaussian norms are not known and the evaluation of the norm may be a challenging problem even in the simplest cases.

In Section 2 below, the sub-Gaussian norm is found for centered Bernoulli random variables. Then the sub-Gaussian norm is evaluated in Section 3 for centered binary random variables, that is, for random variables assuming two values. Using these results, we obtain the exact values of the sub-Gaussian characteristics of empirical distribution functions in Section 4 and exponential bounds for distributions of sums of binary random variables in Section 5 (for both cases, independent and dependent random variables). Exponential bounds for distributions of Bernoulli random variables are considered in Section 6. The bounds obtained for sums of Bernoulli and binary random variables are sharper in some cases than the well-known Bernstein [5] and Hoeffding [14 inequalities. In Section 7 we establish sufficient conditions for an infinite series of centered binary random variables to be sub-Gaussian.

\section{MAin RESUlT}

Let $\beta(p)$ denote a Bernoulli random variable, that is, a random variable assuming the values 1 and 0 with probabilities $p \in[0,1]$ and $q=1-p$, respectively. Along with $\beta(p)$ we consider a centered Bernoulli random variable with parameter $p$ :

$$
\beta^{(0)}(p)=\beta(p)-\mathbf{E} \beta(p)=\beta(p)-p
$$

that assumes the values $q$ and $(-p)$ with probabilities $p$ and $q$, respectively.

The sub-Gaussian norm of the random variable $\beta^{(0)}(p)$ is established in the following theorem.

Theorem 2.1. Let $\beta^{(0)}(p)$ be a centered Bernoulli random variable with parameter $p \in[0,1]$. Then

$$
\tau^{2}\left(\beta^{(0)}(p)\right)=K(p)
$$


where

$$
K(p)= \begin{cases}0, & p \in\{0,1\} \\ \frac{1}{4}, \quad & p=\frac{1}{2} ; \\ \frac{p-q}{2(\ln p-\ln q)}, & p \in(0,1) \backslash\left\{\frac{1}{2}\right\} .\end{cases}
$$

We obtain some properties of the function $K$ defined by equality (4) prior to the proof of Theorem 2.1.

Lemma 2.1. The function $K$ defined by equality (4) has the following properties.

(K1) $K$ is symmetric about the point $p=\frac{1}{2}$ and is positive in the interval $(0,1)$;

(K2) $K$ is continuous in the interval $[0,1]$;

(K3) $K$ increases in the interval $\left[0, \frac{1}{2}\right]$ and decreases in the interval $\left[\frac{1}{2}, 1\right]$;

$$
\max _{p \in[0,1]} K(p)=K\left(\frac{1}{2}\right)=\frac{1}{4}
$$

(K5) $K$ is a convex function in the interval $[0,1]$;

(K6) for every $p \in(0,1) \backslash\left\{\frac{1}{2}\right\}$,

$$
\begin{aligned}
\frac{|p-q|}{|\ln (p \wedge q)|} & =\frac{|p-q|}{\ln \left(\frac{1}{p \wedge q}\right)}<2 K(p)=\frac{|p-q|}{\ln \left(\frac{1}{p \wedge q}-1\right)} \\
& <\frac{|p-q|}{|\ln (2 p \wedge 2 q)|}=\frac{|p-q|}{|\ln (p \wedge q)|-\ln 2}<\frac{1}{|\ln (p \wedge q)|-\ln 2},
\end{aligned}
$$

where $p \wedge q=\min \{p, q\}$;

$$
\lim _{p \downarrow 0} K(p)|\ln p|=\lim _{p \uparrow 1} K(p)|\ln (1-p)|=\frac{1}{2},
$$

that is, $K(p) \sim 1 /|2 \ln p|$ as $p \downarrow 0$ and $K(p) \sim 1 /|2 \ln (1-p)|$ as $p \uparrow 1$;

(K8) for every $p \in(0,1) \backslash\left\{\frac{1}{2}\right\}$,

$$
K(p)=\frac{q-p}{2 H^{\prime}(p)}
$$

where

$$
H(p)=-p \ln p-q \ln q
$$

is the entropy of the Bernoulli random variable $\beta(p)$ and where $H^{\prime}(p)$ is the derivative of the function $H(p)$.

Proof. Since $K(p)=K(1-p)$ for all $p \in[0,1]$, the function $K$ is symmetric about the point $p=\frac{1}{2}$ in the interval $[0,1]$. Moreover, if $p \in(0,1) \backslash\left\{\frac{1}{2}\right\}$, then

$$
K(p)=\frac{\left|p-\frac{1}{2}\right|}{\ln \left(\frac{1}{2}+\left|p-\frac{1}{2}\right|\right)-\ln \left(\frac{1}{2}-\left|p-\frac{1}{2}\right|\right)} .
$$

The positivity of the function $K$ in the interval $(0,1)$ follows from definition (4).

The continuity of the function $K$ for $p \in(0,1) \backslash\left\{\frac{1}{2}\right\}$ is clear from the definition (4). Now we check that $K$ is continuous at the points $p=0, p=1$, and $p=\frac{1}{2}$. Since

$$
\begin{aligned}
& \lim _{p \downarrow \frac{1}{2}} K(p)=\lim _{p \downarrow \frac{1}{2}} \frac{\left|p-\frac{1}{2}\right|}{\ln \left(\frac{1}{2}+\left|p-\frac{1}{2}\right|\right)-\ln \left(\frac{1}{2}-\left|p-\frac{1}{2}\right|\right)}=\frac{1}{4}, \\
& \lim _{p \uparrow \frac{1}{2}} K(p)=\lim _{p \uparrow \frac{1}{2}} \frac{\left|p-\frac{1}{2}\right|}{\ln \left(\frac{1}{2}+\left|p-\frac{1}{2}\right|\right)-\ln \left(\frac{1}{2}-\left|p-\frac{1}{2}\right|\right)}=\frac{1}{4},
\end{aligned}
$$


and $K\left(\frac{1}{2}\right)=\frac{1}{4}$, the function $K$ is continuous at the point $p=\frac{1}{2}$. It is continuous at the points $p=0$ and $p=1$, too, since

$$
\begin{aligned}
& \lim _{p \downarrow 0} K(p)=\lim _{p \downarrow 0} \frac{\left|p-\frac{1}{2}\right|}{\ln \left(\frac{1}{2}+\left|p-\frac{1}{2}\right|\right)-\ln \left(\frac{1}{2}-\left|p-\frac{1}{2}\right|\right)}=0, \\
& \lim _{p \uparrow 1} K(p)=\lim _{p \uparrow 1} \frac{\left|p-\frac{1}{2}\right|}{\ln \left(\frac{1}{2}+\left|p-\frac{1}{2}\right|\right)-\ln \left(\frac{1}{2}-\left|p-\frac{1}{2}\right|\right)}=0,
\end{aligned}
$$

and $K(0)=K(1)=0$. Therefore $K$ is continuous throughout in the interval $[0,1]$.

Further, by the Cauchy-Bunyakovskiu inequality,

$$
\ln p-\ln q=\int_{q}^{p} \frac{d u}{u} \leq \sqrt{(p-q) \int_{q}^{p} \frac{d u}{u^{2}}}=\frac{p-q}{\sqrt{p q}} .
$$

Thus

$$
K(p) \geq \frac{\sqrt{p q}}{2}>p q
$$

for all $p \in(0,1) \backslash\left\{\frac{1}{2}\right\}$.

The derivative of the function $K$ in the set $(0,1) \backslash\left\{\frac{1}{2}\right\}$ is given by

$$
K^{\prime}(p)=\frac{p q-K(p)}{p q(\ln p-\ln q)} .
$$

This together with bound (9) implies that $K^{\prime}(p)<0$ for all $p \in(1 / 2,1)$ and $K^{\prime}(p)>0$ for all $p \in(0,1 / 2)$. Hence the function $K$ decreases in the interval $\left(\frac{1}{2}, 1\right]$ and increases in the interval $\left[0, \frac{1}{2}\right)$. Therefore property (K3) is proved, since $K$ is a continuous function in the interval $[0,1]$.

Taking into account equality (4), we prove property (5) and, moreover,

$$
K(p)<\frac{1}{4}, \quad p \neq \frac{1}{2} .
$$

The second derivative of the function $K$ in the set $(0,1) \backslash\left\{\frac{1}{2}\right\}$ is given by

$$
K^{\prime \prime}(p)=\frac{2\left(K(p)-\frac{1}{4}\right)}{p^{2} q^{2}(\ln p-\ln q)^{4}} .
$$

According to bound (10),

$$
K^{\prime \prime}(p)<0, \quad p \in(0,1) \backslash\left\{\frac{1}{2}\right\} .
$$

Thus the continuous function $K$ is convex in the intervals $\left(0, \frac{1}{2}\right)$ and $\left(\frac{1}{2}, 1\right)$, which proves that $K$ is convex in the interval $[0,1]$.

Finally, let $p \in\left(0, \frac{1}{2}\right)$. Then

$$
\frac{|p-q|}{|\ln p|}=\frac{|p-q|}{\ln \left(\frac{1}{p}\right)}<2 K(p)=\frac{|p-q|}{\ln \left(\frac{1}{p}-1\right)}<\frac{|p-q|}{\ln \left(\frac{1}{2 p}\right)}<\frac{|p-q|}{|\ln p|-\ln 2}<\frac{1}{|\ln p|-\ln 2},
$$

whence property (6) follows. In turn, relation (7) follows from (6). Finally, property (8) follows from equality (4), since $H^{\prime}(p)=\ln q-\ln p$ for all $p \in(0,1)$. 
Proof of Theorem 2.1. For every $p \in[0,1]$, the random variable $\beta^{(0)}(p)$ is centered and bounded, whence we conclude that $\beta^{(0)}(p) \in \operatorname{Sub}(\Omega)$ (see [3,9]).

Note that $\beta^{(0)}(p)=0$ almost surely if either $p=0$ or $p=1$, that is, $\tau\left(\beta^{(0)}(p)\right)=0$, which proves (3).

It is well known that $\tau^{2}\left(\beta^{(0)}\left(\frac{1}{2}\right)\right)=\frac{1}{4}$ for $p=\frac{1}{2}$ (see, for example, [3, 16, 17]). Thus equality (3) holds for $p=\frac{1}{2}$, as well.

It remains to prove equality (3) for $p \in(0,1) \backslash\left\{\frac{1}{2}\right\}$. Since

$$
\tau^{2}(\xi)=\sup _{\lambda \neq 0} \frac{\ln \mathrm{E} e^{\lambda \xi}}{\lambda^{2}}
$$

for any sub-Gaussian random variable $\xi$, we conclude that

$$
\tau^{2}\left(\beta^{(0)}(p)\right)=\sup _{\lambda \neq 0} G_{p}(\lambda),
$$

where

$$
G_{p}(\lambda)=\frac{2 \ln \left(p e^{\lambda q}+q e^{-\lambda p}\right)}{\lambda^{2}}, \quad \lambda \in \mathbf{R} \backslash\{0\} .
$$

Therefore Theorem 2.1 follows if

$$
K(p)=\sup _{\lambda \neq 0} G_{p}(\lambda)
$$

for all $p \in(0,1) \backslash\left\{\frac{1}{2}\right\}$.

It is clear that

$$
G_{p}\left(2 \ln \left(\frac{q}{p}\right)\right)=K(p), \quad p \in(0,1) \backslash\left\{\frac{1}{2}\right\} .
$$

Hence it remains to show that

$$
G_{p}(\lambda) \leq K(p)
$$

for all $p \in(0,1) \backslash\left\{\frac{1}{2}\right\}$ and $\lambda \in \mathbf{R} \backslash\{0\}$. The latter property is equivalent to

$$
h_{p}(\lambda) \leq 0,
$$

where

$$
h_{p}(\lambda)=\ln \left(p e^{\lambda}+q\right)-\lambda p-\frac{K(p) \cdot \lambda^{2}}{2} .
$$

To prove inequality (13), consider the derivatives of the function $h_{p}$. A straightforward computation yields

$$
\begin{gathered}
h_{p}^{\prime}(\lambda)=\frac{p e^{\lambda}}{p e^{\lambda}+q}-p-K(p) \cdot \lambda \\
h_{p}^{\prime \prime}(\lambda)=\frac{q p e^{\lambda}}{\left(p e^{\lambda}+q\right)^{2}}-K(p) \text { and } \quad h_{p}^{\prime \prime \prime}(\lambda)=\frac{q p e^{\lambda}\left(q-p e^{\lambda}\right)}{\left(p e^{\lambda}+q\right)^{3}} .
\end{gathered}
$$

The latter equality implies that

(i) $h_{p}^{\prime \prime \prime}(\lambda)=0$ if $\lambda=\ln (q / p)$;

(ii) $h_{p}^{\prime \prime \prime}(\lambda)>0$ if $\lambda<\ln (q / p)$, and

(iii) $h_{p}^{\prime \prime \prime}(\lambda)<0$ if $\lambda>\ln (q / p)$.

This proves the following properties of the function $h$ :
(A) $h_{p}^{\prime \prime}$ increases in the interval $(-\infty, \ln (q / p))$;
(B) $h_{p}^{\prime \prime}$ decreases in the interval $(\ln (q / p), \infty)$;
(C) $h_{p}^{\prime \prime}$ attains the maximum at $\lambda=\ln (q / p)$. 
Moreover, relations (9) and (10) imply that

$$
h_{p}^{\prime \prime}(2 \ln (q / p))=h_{p}^{\prime \prime}(0)=q p-K(p)<0
$$

and

$$
h_{p}^{\prime \prime}(\ln (q / p))=\frac{1}{4}-K(p)>0 .
$$

Below we list some properties of the derivative $h_{p}^{\prime}$ :

$$
\begin{gathered}
h_{p}^{\prime}(0)=0, \\
h_{p}^{\prime}(\ln (q / p))=0 \\
h_{p}^{\prime}(2 \ln (q / p))=0 .
\end{gathered}
$$

The cases $p \in\left(0, \frac{1}{2}\right)$ and $p \in\left(\frac{1}{2}, 1\right)$ are considered separately.

Let $p \in\left(\frac{1}{2}, 1\right)$. Then relations (14), (15), properties (A)-(C), and continuity of the function $h_{p}^{\prime \prime}$ imply

(D) the equality $h_{p}^{\prime}(\lambda)=0$ holds for at most two points $\lambda_{1}$ and $\lambda_{2}$ such that

$$
\lambda_{1} \in\left(2 \ln \frac{q}{p}, \ln \frac{q}{p}\right) \quad \text { and } \quad \lambda_{2} \in\left(\ln \frac{q}{p}, 0\right) .
$$

Properties (A)-(D) imply four more properties of the function $h_{p}^{\prime}$ :

(E) $h_{p}^{\prime}$ increases in the interval $\lambda \in\left(\lambda_{1}, \lambda_{2}\right)$;

(F) $h_{p}^{\prime}$ decreases in the intervals $\lambda \in\left(-\infty, \lambda_{1}\right)$ and $\lambda \in\left(\lambda_{2}, \infty\right)$;

(G) $h_{p}^{\prime}$ attains the minimum at $\lambda=\lambda_{1}$;

(H) $h_{p}^{\prime}$ attains the maximum at $\lambda=\lambda_{2}$.

Since

$$
h_{p}(0)=0
$$

and

$$
h_{p}\left(2 \ln \frac{q}{p}\right)=0,
$$

we are able to analyze the behavior of the function $h_{p}$ in the whole domain of its definition. It follows from properties (18), (20), and (F) that

(I) $h_{p}(\lambda)<0$ and $h_{p}$ increases in the interval $\left(-\infty, 2 \ln \frac{q}{p}\right)$.

In turn, property (F) and equalities (16) and (19) imply that

(II) $h_{p}(\lambda)<0$ and $h_{p}$ decreases in the interval $(0, \infty)$.

Moreover, we derive from properties (E)-(G) and equalities (17)-(18) that

(III) $h_{p}(\lambda)<0$ for $\lambda \in\left(2 \ln \frac{q}{p}, \ln \frac{q}{p}\right)$.

Finally, using properties (E), (F), and (H) together with equalities (16)-(17) we obtain

(IV) $h_{p}(\lambda)<0$ for $\lambda \in\left(\ln \frac{q}{p}, 0\right)$.

Properties (I)-(IV) show that the function $h_{p}$ has local maximums in the interval $\left[2 \ln \frac{q}{p}, 0\right]$ at the points $\lambda=0$ and $\lambda=2 \ln (q / p)$ and a local minimum at the point $\lambda=\ln \frac{q}{p}$. Hence the function $h_{p}$ attains its maximal value at the points $\lambda=0$ and $\lambda=2 \ln \frac{q}{p}$. This implies that inequalities (13) and (12) hold for all $\lambda \in \mathbf{R}$. Therefore if $p \in\left(\frac{1}{2}, 1\right)$, then $K(p)$ is the maximal value of the function $G_{p}$, that is, equality (11) holds. 
Now let $p \in\left(0, \frac{1}{2}\right)$. Since the function $K$ is symmetric about the point $p=\frac{1}{2}$ and

$$
G_{p}(\lambda)=G_{q}(-\lambda), \quad \lambda \in \mathbf{R} \backslash\{0\},
$$

we deduce from the above case that equality (11) holds for $p \in\left(0, \frac{1}{2}\right)$, too.

The proof of Theorem 2.1 is complete.

\section{The SUb-GaUsSian NORM OF A BINARY RANDOM VARIABle}

The result obtained in the preceding section for centered Bernoulli random variables allows us to find the exact values of sub-Gaussian norms of centered binary random variables.

Theorem 3.1. Let $X$ be a random variable that assumes two values $b$ and $a, b>a$, with probabilities $p \in[0,1]$ and $q=1-p$, respectively. Then

$$
\tau^{2}(X-\mathrm{E} X)=(b-a)^{2} K(p),
$$

where $K$ is the function defined in (4).

Proof. Since

$$
\mathrm{E} X=b \cdot p+a \cdot q=(b-a) p+a
$$

we have

$$
\mathrm{P}\{X-\mathrm{E} X=(b-a) q\}=p \quad \text { and } \quad \mathrm{P}\{X-\mathrm{E} X=-(b-a) p\}=q,
$$

that is,

$$
X-\mathrm{E} X=(b-a) \beta^{(0)}(p),
$$

where $\beta^{(0)}(p)$ is a centered Bernoulli random variable with parameter $p$. Thus

$$
\tau(X-\mathrm{E} X)=(b-a) \tau\left(\beta^{(0)}(p)\right) .
$$

Now Theorem 2.1 implies equality (21).

Remark 3.1. Let $X$ be the same binary random variable as in Theorem 3.1. Then $\mathrm{E}(X-\mathrm{E} X)^{2}=(b-a)^{2} p q$. Relations (21) and (9) imply that the equality

$$
\tau^{2}(X-\mathrm{E} X)=\mathrm{E}(X-\mathrm{E} X)^{2}
$$

holds only if either $p=0$ or $p=1$ or $p=\frac{1}{2}$. This means that a centered binary random variable $X-\mathrm{E} X$ is strictly sub-Gaussian only in those three exceptional cases. Moreover, relations (5) and (10) show that $\tau^{2}(X-\mathrm{E} X) \leq(b-a)^{2} / 4$. This inequality becomes an equality only if $p=\frac{1}{2}$.

Let $X_{j}, j=1, \ldots, n$, be binary random variables assuming values $b_{j}$ and $a_{j}, b_{j}>a_{j}$, with probabilities $p_{j} \in[0,1]$ and $q_{j}=1-p_{j}$, respectively. Since $\tau$ is a norm in the space of sub-Gaussian random variables, Theorem 3.1 implies that

$$
\tau\left(\sum_{j=1}^{n}\left(X_{j}-\mathrm{E} X_{j}\right)\right) \leq \sum_{j=1}^{n} \tau\left(X_{j}-\mathrm{E} X_{j}\right)=\sum_{j=1}^{n}\left(b_{j}-a_{j}\right) K^{\frac{1}{2}}\left(p_{j}\right)
$$

and

$$
\tau^{2}\left(\sum_{j=1}^{n}\left(X_{j}-\mathrm{E} X_{j}\right)\right) \leq\left(\sum_{j=1}^{n}\left(b_{j}-a_{j}\right) K^{\frac{1}{2}}\left(p_{j}\right)\right)^{2}
$$

Therefore

$$
\tau^{2}\left(\sum_{j=1}^{n}\left(X_{j}-\mathrm{E} X_{j}\right)\right) \leq n^{2}(b-a)^{2} K(p)
$$


for identically distributed random variables $X_{j}, j=1, \ldots, n$, assuming values $b$ and $a$, $b>a$, with probabilities $p \in[0,1]$ and $q=1-p$, respectively.

If binary random variables $X_{j}, j=1, \ldots, n$, are independent, then one can improve inequalities (22)-(24). Indeed, inequality (1) and Theorem 3.1 imply that

$$
\tau^{2}\left(\sum_{j=1}^{n}\left(X_{j}-\mathrm{E} X_{j}\right)\right) \leq \sum_{j=1}^{n} \tau^{2}\left(X_{j}-\mathrm{E} X_{j}\right)=\sum_{j=1}^{n}\left(b_{j}-a_{j}\right)^{2} K\left(p_{j}\right) .
$$

The following result shows that inequality (1) becomes an equality for identically distributed sub-Gaussian random variables.

Lemma 3.1. Let $\xi_{1}, \ldots, \xi_{n}$ be independent sub-Gaussian random variables being identically distributed with $\xi$. Then

$$
\begin{gathered}
\tau^{2}\left(\sum_{j=1}^{n} \xi_{j}\right)=\sum_{j=1}^{n} \tau^{2}\left(\xi_{j}\right)=n \tau^{2}(\xi) \\
\tau^{2}\left(\frac{1}{\sqrt{n}} \sum_{j=1}^{n} \xi_{j}\right)=\tau^{2}(\xi), \quad \text { and } \quad \tau^{2}\left(\frac{1}{n} \sum_{j=1}^{n} \xi_{j}\right)=\frac{\tau^{2}(\xi)}{n} .
\end{gathered}
$$

Proof. If $S_{n}=\sum_{j=1}^{n} \xi_{j}$, then

$$
\tau^{2}\left(S_{n}\right)=\sup _{\lambda \neq 0} \frac{\ln \mathrm{E} e^{\lambda S_{n}}}{\lambda^{2}}=\sup _{\lambda \neq 0} \frac{\ln \left(\mathrm{E} e^{\lambda \xi}\right)^{n}}{\lambda^{2}}=n \sup _{\lambda \neq 0} \frac{\ln \mathrm{E} e^{\lambda \xi}}{\lambda^{2}}=n \tau^{2}(\xi),
$$

whence (26) follows. Since $\tau$ is a norm, equalities (27) follow from (26).

Remark 3.2. If sub-Gaussian random variables $\xi_{1}, \ldots, \xi_{n}$ are independent, then the first equality in (26) holds if there exists a number $\lambda_{0} \neq 0$ such that

$$
\sup _{\lambda \neq 0} \frac{\ln \mathrm{E} e^{\lambda \xi_{j}}}{\lambda^{2}}=\frac{\ln \mathrm{E} e^{\lambda_{0} \xi_{j}}}{\lambda_{0}^{2}}
$$

for all $j=1, \ldots, n$, or if

$$
\sup _{\lambda \neq 0} \frac{\ln \mathrm{E} e^{\lambda \xi_{j}}}{\lambda^{2}}=\lim _{\lambda \rightarrow 0} \frac{\ln \mathrm{E} e^{\lambda \xi_{j}}}{\lambda^{2}}=\mathrm{E} \xi_{j}^{2}
$$

for all $j=1, \ldots, n$. Note that the preceding conditions are satisfied if, for example, $\xi_{1}, \ldots, \xi_{n}$ are identically distributed as in Lemma 3.1 or are strictly sub-Gaussian random variables.

Consider some corollaries to Theorem 3.1 and Lemma 3.1 .

Corollary 3.1. Let $X_{j}, j=1, \ldots, n$, be independent identically distributed binary random variables assuming values $b$ and $a, b>a$, with probabilities $p \in[0,1]$ and $q=1-p$, respectively. Then

$$
\begin{gathered}
\tau^{2}\left(\sum_{j=1}^{n}\left(X_{j}-\mathrm{E} X_{j}\right)\right)=n(b-a)^{2} K(p), \\
\tau^{2}\left(\frac{1}{\sqrt{n}} \sum_{j=1}^{n}\left(X_{j}-\mathrm{E} X_{j}\right)\right)=K(p), \quad \text { and } \quad \tau^{2}\left(\frac{1}{n} \sum_{j=1}^{n}\left(X_{j}-\mathrm{E} X_{j}\right)\right)=\frac{K(p)}{n} .
\end{gathered}
$$


Corollary 3.2. Let $\beta_{j}(p), j=1, \ldots, n$, be independent identically distributed Bernoulli random variables with parameter $p \in[0,1]$. Then

$$
\begin{gathered}
\tau^{2}\left(\sum_{j=1}^{n}\left(\beta_{j}(p)-p\right)\right)=n K(p) \\
\tau^{2}\left(\frac{1}{\sqrt{n}} \sum_{j=1}^{n}\left(\beta_{j}(p)-p\right)\right)=K(p), \quad \text { and } \quad \tau^{2}\left(\frac{1}{n} \sum_{j=1}^{n} \beta_{j}(p)-p\right)=\frac{K(p)}{n} .
\end{gathered}
$$

\section{Sub-Gaussian Characteristics of Empirical Distribution FunCtions}

Let $\eta_{j}, j=1, \ldots, n$, be independent random variables being identically distributed with a random variable $\eta$ whose distribution function is $F(t)=\mathrm{P}\{\eta<t\}, t \in \mathbf{R}$. We treat the centered empirical distribution function

$$
\Upsilon_{n}(t)=\hat{F}_{n}(t)-F(t)=\frac{1}{n} \sum_{j=1}^{n} \mathbf{1}\left\{\eta_{j}<t\right\}-F(t)=\frac{1}{n} \sum_{j=1}^{n}\left(\mathbf{1}\left\{\eta_{j}<t\right\}-F(t)\right)
$$

as a stochastic process depending on a parameter $t \in \mathbf{R}$. Since $\mathbf{1}\left\{\eta_{j}<t\right\}$ is a Bernoulli random variable with parameter $p(t)=F(t)$ and increments $\mathbf{1}\left\{\eta_{j}<t\right\}-\mathbf{1}\left\{\eta_{j}<s\right\}, s<t$, also are Bernoulli random variables with parameter $p(t, s)=F(t)-F(s)$, Corollary 3.2 and property (K7) of Lemma 2.1 imply the following result.

Theorem 4.1. Let $\eta_{j}, j=1, \ldots, n$, be independent identically distributed random variables with the distribution function $F(t), t \in \mathbf{R}$. Then

$$
\tau^{2}\left(\Upsilon_{n}(t)\right)=\frac{K(F(t))}{n}, \quad \tau^{2}\left(\sqrt{n} \Upsilon_{n}(t)\right)=K(F(t))
$$

and

$$
\begin{gathered}
\tau^{2}\left(\Upsilon_{n}(t)-\Upsilon_{n}(s)\right)=\frac{K(F(t)-F(s))}{n}, \\
\tau^{2}\left(\sqrt{n}\left(\Upsilon_{n}(t)-\Upsilon_{n}(s)\right)\right)=K(F(t)-F(s)) .
\end{gathered}
$$

In addition, if the distribution function $F$ is continuous, then

$$
\tau^{2}\left(\sqrt{n}\left(\Upsilon_{n}(t)-\Upsilon_{n}(s)\right)\right) \sim \frac{1}{2 \ln (F(t)-F(s))}
$$

for all $n \geq 1$ as $(t-s) \rightarrow 0$.

In particular, if $F(t)=t, t \in[0,1]$, that is, if $\eta_{j}, j=1, \ldots, n$, are independent random variables uniformly distributed in the interval $[0,1]$, then

$$
\begin{aligned}
& \tau^{2}\left(\Upsilon_{n}(t)\right)=\frac{K(t)}{n}, \quad \tau^{2}\left(\sqrt{n} \Upsilon_{n}(t)\right)=K(t), \\
& \tau^{2}\left(\Upsilon_{n}(t)-\Upsilon_{n}(s)\right)=\frac{K(t-s)}{n}, \quad \tau^{2}\left(\sqrt{n}\left(\Upsilon_{n}(t)-\Upsilon_{n}(s)\right)\right)=K(t-s)
\end{aligned}
$$

for $0 \leq s<t \leq 1$. In addition,

$$
\tau^{2}\left(\sqrt{n}\left(\Upsilon_{n}(t)-\Upsilon_{n}(s)\right)\right) \sim \frac{1}{2 \ln (t-s)}
$$

as $(t-s) \rightarrow 0$ for all $n \geq 1$. 


\section{EXPONENTIAL BOUNDS FOR DISTRIBUTIONS OF SUMS OF BINARY RANDOM VARIABLES}

Theorem 3.1 implies some exponential bounds for the tails of distributions of sums of binary random variables.

Theorem 5.1. Let $X_{j}, j=1, \ldots, n$, be binary random variables assuming values $b_{j}$ and $a_{j}, b_{j}>a_{j}$, with probabilities $p_{j} \in[0,1]$ and $q_{j}=1-p_{j}$, respectively. Let

$$
S_{n}^{(0)}=\sum_{j=1}^{n}\left(X_{j}-\mathrm{E} X_{j}\right)
$$

and let the function $K$ be defined by (44). Then

(i) for all $x>0$,

$$
\begin{gathered}
\mathrm{P}\left\{S_{n}^{(0)} \geq x\right\} \leq \mathcal{E}\left(x ; p_{1}, \ldots, p_{n}\right), \\
\mathrm{P}\left\{S_{n}^{(0)} \leq-x\right\} \leq \mathcal{E}\left(x ; p_{1}, \ldots, p_{n}\right), \\
\mathrm{P}\left\{\left|S_{n}^{(0)}\right| \geq x\right\} \leq 2 \mathcal{E}\left(x ; p_{1}, \ldots, p_{n}\right),
\end{gathered}
$$

where

$$
\mathcal{E}\left(x ; p_{1}, \ldots, p_{n}\right)=\exp \left\{\frac{-x^{2}}{B\left(p_{1}, \ldots, p_{n}\right)}\right\}
$$

and

$$
B\left(p_{1}, \ldots, p_{n}\right)=2\left(\sum_{j=1}^{n}\left(b_{j}-a_{j}\right) K^{\frac{1}{2}}\left(p_{j}\right)\right)^{2} ;
$$

(ii) if random variables $X_{j}, j=1, \ldots, n$, are independent, then inequalities (28) hold for all $x>0$ with

$$
B\left(p_{1}, \ldots, p_{n}\right)=2 \sum_{j=1}^{n} K\left(p_{j}\right)\left(b_{j}-a_{j}\right)^{2} .
$$

Proof. Statement (i) follows from inequalities (11) and (23). In turn, statement (ii) follows from inequalities (1) and (25).

Remark 5.1. Since $K\left(p_{j}\right)=K\left(q_{j}\right), j=1, \ldots, n$, we conclude that

$$
\mathcal{B}\left(p_{1}, \ldots, p_{n}\right)=\mathcal{B}\left(q_{1}, \ldots, q_{n}\right) .
$$

The Hoeffding inequality is one of the well-known exponential bounds for sums of independent bounded random variables (see [14]). According to the Hoeffding inequality

$$
\mathrm{P}\left\{\sum_{j=1}^{n}\left(X_{j}-\mathrm{E} X_{j}\right) \geq x\right\} \leq \exp \left\{\frac{-2 x^{2}}{\sum_{j=1}^{n}\left(b_{j}-a_{j}\right)^{2}}\right\}
$$

for all $x>0$, where $X_{j}, j=1, \ldots, n$, are independent random variables bounded from below and from above, that is, they are such that

$$
\mathrm{P}\left\{a_{j} \leq X_{j} \leq b_{j}\right\}=1, \quad j=1, \ldots, n .
$$

Since $K(p)<\frac{1}{4}$ for any $p \neq \frac{1}{2}$, we see from statement (ii) of Theorem 5.1 for independent random variables that the first inequality in (28) is sharper than inequality (29). Note, however, that the right-hand sides of these inequalities coincide if $p_{j}=\frac{1}{2}$, $j=1, \ldots, n$. But if $p_{j}$ are close to either 0 or 1 , then (28) becomes much sharper than Hoeffding's inequality (29). 


\section{EXPONENTIAL BOUNDS FOR DISTRIBUTIONS OF SUMS OF BERNOULLI RANDOM VARIABLES}

Theorem 5.1 for Bernoulli random variables reads as follows.

Theorem 6.1. Let $\beta_{j}\left(p_{j}\right), j=1, \ldots, n$, be Bernoulli random variables,

$$
S_{n}=\sum_{j=1}^{n} \beta_{j}\left(p_{j}\right), \quad S_{n}^{(0)}=S_{n}-\sum_{j=1}^{n} p_{j},
$$

and let the function $K$ be defined in (4). Then

(i) for all $x>0$, inequalities (28) hold with

$$
B\left(p_{1}, \ldots, p_{n}\right)=2\left(\sum_{j=1}^{n} K^{\frac{1}{2}}\left(p_{j}\right)\right)^{2} ;
$$

(ii) if random variables $\beta_{j}\left(p_{j}\right), j=1, \ldots, n$, are independent, then (28) holds for all $x>0$ with

$$
B\left(p_{1}, \ldots, p_{n}\right)=2 \sum_{j=1}^{n} K\left(p_{j}\right) .
$$

Next we consider a corollary of Theorem 6.1 for identically distributed Bernoulli random variables.

Corollary 6.1. Let $\beta_{j}(p), j=1, \ldots, n$, be identically distributed Bernoulli random variables,

$$
S_{n}=\sum_{j=1}^{n} \beta_{j}(p),
$$

and let the function $K$ be defined by (4). Then

(i) for all $x>0$,

$$
\begin{gathered}
\mathrm{P}\left\{S_{n}-n p \geq x\right\} \leq \exp \left\{\frac{-x^{2}}{2 n^{2} K(p)}\right\}, \\
\mathrm{P}\left\{S_{n}-n p \leq-x\right\} \leq \exp \left\{\frac{-x^{2}}{2 n^{2} K(p)}\right\}, \\
\mathrm{P}\left\{\left|S_{n}-n p\right| \geq x\right\} \leq 2 \exp \left\{\frac{-x^{2}}{2 n^{2} K(p)}\right\} .
\end{gathered}
$$

In particular,

$$
\mathrm{P}\left\{\left|\frac{S_{n}}{n}-p\right| \geq x\right\} \leq 2 \exp \left\{\frac{-x^{2}}{2 K(p)}\right\}
$$

for all $x>0$;

(ii) if random variables $\beta_{j}(p), j=1, \ldots, n$, are independent, then

$$
\begin{gathered}
\mathrm{P}\left\{S_{n}-n p \geq x\right\} \leq \exp \left\{\frac{-x^{2}}{2 n K(p)}\right\}, \\
\mathrm{P}\left\{S_{n}-n p \leq-x\right\} \leq \exp \left\{\frac{-x^{2}}{2 n K(p)}\right\}, \\
\mathrm{P}\left\{\left|S_{n}-n p\right| \geq x\right\} \leq 2 \exp \left\{\frac{-x^{2}}{2 n K(p)}\right\}
\end{gathered}
$$


for all $x>0$; in particular,

$$
\mathrm{P}\left\{\left|\frac{S_{n}}{n}-p\right| \geq x\right\} \leq 2 \exp \left\{\frac{-n x^{2}}{2 K(p)}\right\}
$$

for all $x>0$.

Example 6.1. Let $\beta_{j}(p), j=1, \ldots, n$, be identically distributed Bernoulli random variables and let $p \in\left(0, \frac{1}{2}\right)$. Then

(i) for all $x>0$,

$$
\mathrm{P}\left\{\frac{S_{n}}{n}-p \geq x\right\} \leq 2 \exp \left\{\frac{-x^{2} \ln \left(\frac{1-p}{p}\right)}{1-2 p}\right\}
$$

(ii) if random variables $\beta_{j}(p), j=1, \ldots, n$, are independent, then

$$
\mathrm{P}\left\{\left|\frac{S_{n}}{n}-p\right| \geq x\right\} \leq 2 \exp \left\{\frac{-n x^{2} \ln \left(\frac{1-p}{p}\right)}{1-2 p}\right\}
$$

for all $x>0$.

Note that the latter result coincides with a corresponding Hoeffding inequality obtained in the paper [14].

The bounds in Theorems 5.1 and 6.1 correspond to the well-known large deviation inequalities if random variables are independent and identically distributed (see, for example, [14,15]). Note however that the bounds in Theorems 5.1 and 6.1 are sometimes less precise than the large deviation inequalities. On the other hand, our bounds in Theorems 5.1 and 6.1 provide additional information about the deviations if the random variables are dependent or independent but nonidentically distributed.

Every sequence of $n$ Bernoulli trials with the success probability $p_{j}$ corresponds to a sequence of Bernoulli random variables $\beta_{j}\left(p_{j}\right), j=1, \ldots, n$, whose sum $\sum_{j=1}^{n} \beta_{j}\left(p_{j}\right)$ equals the total number of successes in $n$ Bernoulli trials. Note that the Bernoulli trials are dependent (independent) if and only if the corresponding Bernoulli random variables are dependent (independent). The inequalities in Theorem 6.1 written in terms of Bernoulli trials provide the exponential bounds for the distribution of the total number of successes for independent or dependent Bernoulli trials as well as in the homogeneous or nonhomogeneous case and correspond to some known estimates; see [5, 6, 10, 11, 15].

\section{SERIES OF BINARY RANDOM VARIABLES}

The following result contains sufficient conditions for an infinite series of centered binary random variables to be sub-Gaussian.

Theorem 7.1. Let $X_{j}, j \geq 1$, be a sequence of binary random variables assuming values $b_{j}$ and $a_{j}, b_{j}>a_{j}$, with probabilities $p_{j} \in[0,1]$ and $q_{j}=1-p_{j}$, respectively. Let the function $K$ be defined as in (4). Then

(i) if

$$
B_{\infty}\left(\left\{p_{j}\right\}\right)=\left(\sum_{j=1}^{\infty}\left(b_{j}-a_{j}\right) K^{\frac{1}{2}}\left(p_{j}\right)\right)^{2}<\infty,
$$

then the series $\sum_{j=1}^{\infty}\left(X_{j}-\mathrm{E} X_{j}\right)$ converges almost surely and in the space $\operatorname{Sub}(\Omega)$. Moreover, the sum of this series $S_{\infty}^{(0)}$ is a sub-Gaussian random variable such that 
$\tau^{2}\left(S_{\infty}^{(0)}\right) \leq B_{\infty}\left(\left\{p_{j}\right\}\right)$ and

$$
\begin{gathered}
\mathrm{P}\left\{S_{\infty}^{(0)} \geq x\right\} \leq \exp \left\{\frac{-x^{2}}{B_{\infty}\left(\left\{p_{j}\right\}\right)}\right\}, \\
\mathrm{P}\left\{S_{\infty}^{(0)} \leq-x\right\} \leq \exp \left\{\frac{-x^{2}}{B_{\infty}\left(\left\{p_{j}\right\}\right)}\right\}, \\
\mathrm{P}\left\{\left|\left(S_{\infty}^{(0)}\right)\right| \geq x\right\} \leq 2 \exp \left\{\frac{-x^{2}}{B_{\infty}\left(\left\{p_{j}\right\}\right)}\right\}
\end{gathered}
$$

for all $x>0$;

(ii) if the random variables $\left(X_{j}, j \geq 1\right)$ are independent and

$$
B_{\infty}\left(\left\{p_{j}\right\}\right)=\sum_{j=1}^{\infty}\left(b_{j}-a_{j}\right)^{2} K\left(p_{j}\right)<\infty
$$

then statement (i) holds with $B_{\infty}\left(\left\{p_{j}\right\}\right)$ defined by (32) instead of (30).

Proof. Using condition (30) we deduce from Theorem 3.1 that

$$
\sum_{j=1}^{\infty} \mathrm{E}\left|X_{j}-\mathrm{E} X_{j}\right| \leq \sum_{j=1}^{\infty} \tau\left(X_{j}-\mathrm{E} X_{j}\right)=\sum_{j=1}^{\infty}\left(b_{j}-a_{j}\right) K^{\frac{1}{2}}\left(p_{j}\right)<\infty .
$$

Thus both series $\sum_{j=1}^{\infty}\left|X_{j}-\mathrm{E} X_{j}\right|$ and $\sum_{j=1}^{\infty}\left(X_{j}-\mathrm{E} X_{j}\right)$ converge almost surely. Since $\tau$ is a norm in the space $\operatorname{Sub}(\Omega)$ and since $\operatorname{Sub}(\Omega)$ is a Banach space with respect to this norm, the series $\sum_{j=1}^{\infty}\left(X_{j}-\mathrm{E} X_{j}\right)$ converges in the space $\operatorname{Sub}(\Omega)$ and its $\operatorname{sum} S_{\infty}^{(0)}$ is a sub-Gaussian random variable such that $\tau^{2}\left(S_{\infty}^{(0)}\right) \leq B_{\infty}\left(\left\{p_{j}\right\}\right)$, where $B_{\infty}\left(\left\{p_{j}\right\}\right)$ is defined by (30). This together with (11) implies (31). Statement (i) of Theorem [7.1] is proved.

To prove statement (ii) assume that the random variables $X_{j}, j \geq 1$, are independent. Note that condition (32) implies

$$
\sum_{j=1}^{\infty} \mathrm{E}\left(X_{j}-\mathrm{E} X_{j}\right)^{2} \leq \sum_{j=1}^{\infty} \tau^{2}\left(X_{j}-\mathrm{E} X_{j}\right)=\sum_{j=1}^{\infty}\left(b_{j}-a_{j}\right)^{2} K\left(p_{j}\right)<\infty .
$$

Thus the series $\sum_{j=1}^{\infty}\left(X_{j}-\mathrm{E} X_{j}\right)$ converges almost surely. Moreover, relation (25) implies that

$$
\tau\left(\sum_{j=n}^{n+m}\left(X_{j}-\mathrm{E} X_{j}\right)\right)^{2} \leq \sum_{j=n}^{n+m} \tau^{2}\left(X_{j}-\mathrm{E} X_{j}\right)=\sum_{j=n}^{n+m}\left(b_{j}-a_{j}\right)^{2} K\left(p_{j}\right)
$$

for all $n, m \geq 1$. Therefore condition (32) implies that the sequence of partial sums of the series $\sum_{j=1}^{\infty}\left(X_{j}-\mathrm{E} X_{j}\right)$ is a Cauchy sequence in the Banach space $\operatorname{Sub}(\Omega)$. This means that the series converges in the space $\operatorname{Sub}(\Omega)$ and its sum $S_{\infty}^{(0)}$ is a sub-Gaussian random variable such that $\tau^{2}\left(S_{\infty}^{(0)}\right) \leq B_{\infty}\left(\left\{p_{j}\right\}\right)$, where $B_{\infty}\left(\left\{p_{j}\right\}\right)$ is defined by equality (32). Relation (11) completes the proof of statement (ii).

Remark 7.1. Let

$$
0<\liminf _{n \rightarrow \infty}\left(b_{j}-a_{j}\right) \text { and } \quad \limsup _{n \rightarrow \infty}\left(b_{j}-a_{j}\right)<\infty .
$$

According to properties (K6) and (K7) of Lemma 2.1.

(i) condition (30) holds if and only if

$$
\sum_{j=1}^{\infty} \frac{1}{\sqrt{\left|\ln \left(p_{j} \wedge q_{j}\right)\right|}}<\infty
$$


moreover,

$$
B_{\infty}\left(\left\{p_{j}\right\}\right) \leq\left(\sum_{j=1}^{\infty} \frac{b_{j}-a_{j}}{\sqrt{\left|\ln \left(p_{j} \wedge q_{j}\right)\right|-\ln 2}}\right)^{2} ;
$$

(ii) condition (32) holds if and only if

$$
\sum_{j=1}^{\infty} \frac{1}{\left|\ln \left(p_{j} \wedge q_{j}\right)\right|}<\infty
$$

moreover,

$$
B_{\infty}\left(\left\{p_{j}\right\}\right) \leq \sum_{j=1}^{\infty} \frac{\left(b_{j}-a_{j}\right)^{2}}{\left|\ln \left(p_{j} \wedge q_{j}\right)\right|-\ln 2} .
$$

Example 7.1. Let $d=b_{j}-a_{j}>0, j \geq 1$, and let

$$
p_{j}=2^{-(j+1)^{\alpha}}, \quad j \geq 1,
$$

where $\alpha>1$. Then

$$
A_{\alpha}=\sum_{j=1}^{\infty} p_{j}=\sum_{j=1}^{\infty} 2^{-(j+1)^{\alpha}}<\infty
$$

Moreover if $\alpha>2$, then

$$
\sum_{j=1}^{\infty} \frac{1}{\sqrt{\left|\ln \left(p_{j} \wedge q_{j}\right)\right|}}=\left(\sqrt{\log _{2} e}\right) \sum_{j=1}^{\infty} \frac{1}{(j+1)^{\alpha / 2}}<\infty,
$$

while if $\alpha>1$, then

$$
\sum_{j=1}^{\infty} \frac{1}{\left|\ln \left(p_{j} \wedge q_{j}\right)\right|}=\left(\log _{2} e\right) \sum_{j=1}^{\infty} \frac{1}{(j+1)^{\alpha}}<\infty .
$$

Therefore if $\alpha>2$, then conditions (30) and (32) hold (see Remark 7.1); otherwise, that is, if $\alpha \in(1,2]$, then only condition (32) holds.

Theorem [7.1, Remark 7.1, and Example 7.1 imply the following assertion.

Corollary 7.1. Let the success probabilities $p_{j}, j \geq 1$, for an infinite sequence of independent Bernoulli trials satisfy relation (33) and let $S_{\infty}$ denote the total number of successes in this sequence. Then $\mathrm{E} S_{\infty}=A_{\alpha}$ and $\left(S_{\infty}-A_{\alpha}\right)$ is a sub-Gaussian random variable such that

$$
\tau^{2}\left(S_{\infty}-A_{\alpha}\right) \leq\left(\log _{2} e\right) \sum_{j=1}^{\infty} \frac{1}{j^{\alpha}}
$$

In what follows we consider an infinite non-homogeneous sequence of independent Bernoulli trials with success probabilities $p_{j}, j \geq 1$. We assume that the series $\sum_{j=1}^{\infty} p_{j}$ diverges but the series $\sum_{j=1}^{\infty}\left(\beta_{j}\left(p_{j}\right)-p_{j}\right)$ converges almost surely and in the space $\operatorname{Sub}(\Omega)$. Such models naturally appear as a result of "small perturbations" of some degenerate sequences of Bernoulli trials which we are going to describe below.

Let a "nonrandom" sequence of Bernoulli trials correspond to the success probabilities $\mathfrak{p}_{j}, j \geq 1$, and let

$$
\mathfrak{p}_{j}=1 \quad \text { or } \quad \mathfrak{p}_{j}=0
$$

for all $j \geq 1$. This means that the results of Bernoulli trials are nonrandom and the number of successes in $n$ trials is a nonrandom variable

$$
V_{n}=\sum_{j=1}^{n} \mathfrak{p}_{j}, \quad n \geq 1 .
$$


Further let

$$
\sum_{j=1}^{\infty} \mathfrak{p}_{j}=\infty
$$

that is, $V_{n} \rightarrow \infty$ as $n \rightarrow \infty$. It is possible (but it is not always the case) that

$$
\sum_{j=1}^{\infty}\left(1-\mathfrak{p}_{j}\right)=\infty
$$

that is, $n-V_{n} \rightarrow \infty$ as $n \rightarrow \infty$.

Assume that, after "small perturbations", the success probabilities $\mathfrak{p}_{j}, j \geq 1$, transform into $p_{j}, j \geq 1$, such that

$$
\left|\mathfrak{p}_{j}-p_{j}\right|=\delta_{j} \in\left(0, \frac{1}{2}\right), \quad j \geq 1 .
$$

As a result, one observes the random variables $S_{n}=\sum_{j=1}^{n} \beta_{j}\left(p_{j}\right)$ instead of the deterministic sequence of successes $V_{n}, n \geq 1$. The following result shows that if the perturbations $\delta_{j}, j \geq 1$, are small, then the error $Z_{n}=V_{n}-S_{n}, n \geq 1$, becomes sub-Gaussian.

Corollary 7.2. Let the success probabilities $p_{j}, j \geq 1$, for an infinite sequence of Bernoulli trials be such that relations (34)-(35) hold. Let the function $K$ be defined by (4). Then

(i) if

$$
\sum_{j=1}^{\infty} K^{\frac{1}{2}}\left(\delta_{j}\right)<\infty
$$

then the sequence $Z_{n}=V_{n}-S_{n}, n \geq 1$, converges to the limit

$$
Z_{\infty}=\Delta_{\infty}+S_{\infty}^{(0)}
$$

where

$$
\Delta_{\infty}=\sum_{j=1}^{\infty} \delta_{j}<\infty
$$

is the deterministic component of the limit and where

$$
S_{\infty}^{(0)}=\sum_{j=1}^{\infty}\left(p_{j}-\beta_{j}\left(p_{j}\right)\right)
$$

is the random component of the limit such that the series in (37) converges almost surely and in the space $\operatorname{Sub}(\Omega)$. Moreover,

$$
\tau^{2}\left(S_{\infty}^{(0)}\right) \leq\left(\sum_{j=1}^{\infty} K^{\frac{1}{2}}\left(\delta_{j}\right)\right)^{2} ;
$$

(ii) if the Bernoulli trials are independent and

$$
\sum_{j=1}^{\infty} K\left(\delta_{j}\right)<\infty
$$

then statement (i) holds with the difference that the series in (37) consists of independent centered Bernoulli random variables and

$$
\tau^{2}\left(Z_{\infty}\right) \leq \sum_{j=1}^{\infty} K\left(\delta_{j}\right)
$$


Proof. Conditions (34) and (35) imply that $\delta_{j}=p_{j} \wedge\left(1-p_{j}\right), j \geq 1$. Then if conditions (36) and (39) hold, then both series

$$
\sum_{j=1}^{\infty} K^{\frac{1}{2}}\left(p_{j}\right) \text { and } \sum_{j=1}^{\infty} K\left(p_{j}\right)
$$

converge by property (K1) of Lemma 2.1. Thus the series

$$
\sum_{j=1}^{\infty}\left(p_{j}-\beta_{j}\left(p_{j}\right)\right)
$$

converges and inequalities (38) and (40) in statements (i) and (ii) follow from Theorem 7.1. Since

$$
\delta_{j}=p_{j} \wedge\left(1-p_{j}\right) \leq 2 p_{j}\left(1-p_{j}\right) \leq 2 K\left(p_{j}\right) \leq 2 K^{\frac{1}{2}}\left(p_{j}\right)
$$

(see (9)), the convergence of either of the two series

$$
\sum_{j=1}^{\infty} K^{\frac{1}{2}}\left(p_{j}\right) \quad \text { or } \quad \sum_{j=1}^{\infty} K\left(p_{j}\right)
$$

implies the convergence of the series $\sum_{j=1}^{\infty} \delta_{j}$.

The proof of Corollary 7.2 is complete.

\section{Concluding Remarks}

The exact values of the sub-Gaussian norms of Bernoulli and binary random variables are obtained in this paper. These allow us to find the exact values of the sub-Gaussian norms for sums of independent identically distributed binary random variables and for the centered empirical distribution function and its increments. Using these results, exponential bounds are established for the distributions of sums of centered binary random variables (in particular, for sums of Bernoulli random variables). The exponential bounds are valid for both cases of independent and dependent random variables and generalize some known inequalities. The results of this paper can be useful for studies of asymptotic properties of sums and infinite series of binary random variables.

\section{BIBLIOGRAPHY}

[1] V. V. Buldygin, Skhodimost sluchainykh elementov v topologicheskikh prostranstvakh, "Naukova Dumka", Kiev, 1980 (Russian). MR734899(84m:60011)

[2] V. V. Buldygin and Ju. V. Kozačenko, Sub-Gaussian random variables, Ukrain. Mat. Zh. 32 (1980), no. 6, 723-730 (Russian). MR598605 (82d:60023)

[3] V. V. Buldygin and Yu. V. Kozachenko, Metric characterization of random variables and random processes, Translations of Mathematical Monographs, vol. 188, American Mathematical Society, Providence, RI, 2000. Translated from the 1998 Russian original by V. Zaiats. MR,1743716 (2001g:60089)

[4] V. V. Buldygin and E. D. Pechuk, Inequalities for the distributions of functionals of sub-Gaussian vectors, Teor. Imovirnost. Matem. Statist. 80 (2009), 23-33; English transl. in Theor. Probability and Math. Statist. 80 (2010), 25-36.

[5] S. N. Bernstein, On a modification of Chebyshev's inequality and of the error formula of Laplace, Uchenye Zapiski Nauch.-Issled. Kaf. Ukraine, Sect. Math. (1924), no. 1, 38-48. (Russian)

[6] S. N. Bernštel̆n, Sobranie sochinenii. Tom IV: Teoriya veroyatnostei. Matematicheskaya statistika. 1911-1946, Izdat. "Nauka", Moscow, 1964 (Russian). MR.0169758 (30 \#2)

[7] N. N. Vakhaniya, V. V. Kvaratskheliya, and V. I. Tarieladze, Weakly sub-Gaussian random elements in Banach spaces, Ukraïn. Mat. Zh. 57 (2005), no. 9, 1187-1208, DOI 10.1007/s11253-006-0003-y (Russian, with English and Ukrainian summaries); English transl., Ukrainian Math. J. 57 (2005), no. 9, 1387-1412. MR2216040 (2007c:60006)

[8] Yu. V. Kozachenko, Sufficient conditions for the continuity with probability one of sub-Gaussian stochastic processes, Dopovidi Academy of Science of Ukraine, 2 (1968), 113-115. (Ukrainian) 
[9] E. I. Ostrovskiu, Exponential estimates of the distribution of the maximum of a non-Gaussian random field, Teor. Veroyatnost. i Primenen. 35 (1990), no. 3, 482-493, DOI 10.1137/1135068 (Russian); English transl., Theory Probab. Appl. 35 (1990), no. 3, 487-499 (1991). MR1091204 (92f:60086)

[10] V. V. Petrov, A generalization and sharpening of Bernšteı̆n's inequalities, Vestnik Leningrad. Univ. 22 (1967), no. 19, 63-68 (Russian, with English summary). MR.0219111 (36 \#2194)

[11] Valentin V. Petrov, Limit theorems of probability theory, Oxford Studies in Probability, vol. 4, The Clarendon Press Oxford University Press, New York, 1995. Sequences of independent random variables; Oxford Science Publications. MR.1353441 (96h:60048)

[12] Herman Chernoff, A measure of asymptotic efficiency for tests of a hypothesis based on the sum of observations, Ann. Math. Statistics 23 (1952), 493-507. MR0057518 (15,241c)

[13] R. Giuliano Antonini and Yu. V. Kozachenko, A note on the asymptotic behavior of sequences of generalized subGaussian random vectors, Random Oper. Stochastic Equations 13 (2005), no. 1, 39-52, DOI 10.1163/1569397053300900. MR2130246 (2006b:60020)

[14] Wassily Hoeffding, Probability inequalities for sums of bounded random variables, J. Amer. Statist. Assoc. 58 (1963), 13-30. MR0144363 (26 \#1908)

[15] Masashi Okamoto, Some inequalities relating to the partial sum of binomial probabilities, Ann. Inst. Statist. Math. 10 (1958), 29-35. MR0099733 (20 \#6171)

[16] J.-P. Kahane, Propriétés locales des fonctions à séries de Fourier aléatoires, Studia Math. 19 (1960), 1-25 (French). MR0117506 (22 \#8285)

[17] Jean-Pierre Kahane, Some random series of functions, D. C. Heath and Co. Raytheon Education Co., Lexington, Mass., 1968. MR0254888 (40 \#8095)

Department of Mathematical Analysis and Probability Theory, National Technical University of Ukraine "KPI", Peremogy Avenue, 37, Kyiv 03056, Ukraine

E-mail address: matan@kpi.ua

Department of Mathematical Analysis and Probability Theory, National Technical University of Ukraine "KPI", Peremogy Avenue, 37, Kyiv 03056, Ukraine

E-mail address: matan@kpi.ua

Received 10/OCT/2011

Translated by N. SEMENOV 